\title{
Using Cepheids to determine the galactic abundance gradient
}

\section{First results for the outer disc ${ }^{\star} \star \star$}

\author{
S. M. Andrievsky ${ }^{1,2}$, V. V. Kovtyukh, ${ }^{1,2}$, R. E. Luck ${ }^{3}$, J. R. D. Lépine ${ }^{4}$, W. J. Maciel ${ }^{4}$, and Yu. V. Beletsky ${ }^{1,2}$ \\ 1 Department of Astronomy, Odessa State University, Shevchenko Park, 65014 Odessa, Ukraine \\ e-mail: val@deneb.odessa.ua; adelante@ukr.net \\ 2 Odessa Astronomical Observatory and Isaac Newton Institute of Chile, Odessa Branch, Ukraine \\ 3 Department of Astronomy, Case Western Reserve University, 10900 Euclid Avenue, Cleveland, OH 44106-7215, USA \\ e-mail: luck@fafnir.astr.cwru.edu \\ ${ }^{4}$ Instituto Astronômico e Geofísico, Universidade de São Paulo, Rua do Matão 1226, São Paulo SP, Brazil \\ e-mail: jacques@astro.iag.usp.br; maciel@astro.iag.usp.br
}

Received 12 April 2002 / Accepted 25 June 2002

\begin{abstract}
As a continuation of our previous work, which concerned the radial abundance distribution in the galactic disc over the distances 4-10 kpc this paper presents the first results on the metallicity in the outer disc $\left(R_{\mathrm{G}}>10 \mathrm{kpc}\right)$. Based on highresolution spectra obtained for 19 distant Cepheids we sampled galactocentric distances from 10 to $12 \mathrm{kpc}$. Combined with the results of our previous work on the inner and middle parts of the galactic disc, the present data enable one to study the structure of the radial abundance distribution over a large baseline. In particular, we find indications of a discontinuity in the radial abundance distribution for iron as well as a number of the other elements. The discontinuity is seen at a galactocentric distance $R_{\mathrm{G}}=10 \mathrm{kpc}$. This finding supports the results reported earlier by Twarog et al. (1997).
\end{abstract}

Key words. stars: abundances - stars: cepheids - galaxy: abundances - galaxy: evolution

\section{Introduction}

As shown in our previous papers (Andrievsky et al. 2002a Paper I, 2002b - Paper II) the radial abundance distribution within the region of galactocentric distances from 4 to $10 \mathrm{kpc}$ is best described by two distinct zones. One of them (inner: $\left.4.0 \mathrm{kpc}<R_{\mathrm{G}}<6.5 \mathrm{kpc}\right)$ is characterized by a rather steep gradient, while in the mid part of galactic disc $\left(6.5 \mathrm{kpc}<R_{\mathrm{G}}<\right.$ $10.0 \mathrm{kpc}$ ), the distribution is essentially flat (e.g. for iron the gradient is $\mathrm{d}[\mathrm{Fe} / \mathrm{H}] / \mathrm{d} R_{\mathrm{G}} \approx-0.03 \mathrm{dex} / \mathrm{kpc}$ ).

As discussed in Papers I and II, such a bimodal character in the distribution may result from the combined effect of the radial gas flow in the disc and the radial distribution of the star formation rate. We note here that there are conflicting models of the galactic structure, and that possibly the metallicity gradients can help to decide which are the more likely ones. According to Sevenster (1999a, 1999b) and others (see references in Paper I) the bar extends its influence to a corotation radius at about $4-6 \mathrm{kpc}$. In contrast, according to Amaral \& Lépine (1997) and others, the spiral arms extend

Send offprint requests to: S. M. Andrievsky,

e-mail: scan@deneb.odessa.ua

* Based on spectra collected at ESO - La Silla, Chile.

$\star \star$ Table A1 is only available in electronic form at the CDS via anonymous ftp to cdsarc.u-strasbg.fr $(130.79 .128 .5)$ or via http://cdsweb.u-strasbg.fr/cgi-bin/qcat?J/A+A/392/491 from the Inner Lindblad Resonance which is at about $2.5 \mathrm{kpc}$, to the Outer Lindblad Resonance, at about $12 \mathrm{kpc}$, and the corotation of the spiral pattern is close to the solar galactic orbit. In the vicinity of a bar we expect to see elongated orbits of stars, and consequently, a small metallicity gradient. On the other hand, according to Lépine et al. (2001) an interaction between the gas and spiral waves in the disc forces the gas to flow in opposite directions inside and outside the Galactic corotation annulus. This mechanism produces a cleaning effect in the middle part of the disc and consequently a flattening of the metallicity distribution. At the same time, a decreased star formation rate in the vicinity of the galactic co-rotation, where the relative velocity of the spiral arms and of the gas passing through these arms is small, should also result in some decrease in the abundances.

As the next step of this study we have begun to investigate the radial abundance distribution in the outer disc. The region of primary interest is at a galactocentric radius $R_{\mathrm{G}} \approx 10 \mathrm{kpc}$, where according to Twarog et al. (1997) there exists a discontinuity in the metallicity distribution. Such a discontinuity can be suspected from earlier works of Janes (1979), Panagia \& Tosi (1981), and Friel (1995). However, Twarog et al. (1997) were the first to clearly stress this result. Twarog et al. used photometic metallicities (interpreted to imply $[\mathrm{Fe} / \mathrm{H}]$ ) for a large sample of open clusters, and they found that galactic disc 
breaks into two distinct zones. Between $R_{\mathrm{G}} \approx 6.5-10.0 \mathrm{kpc}$ they found a mean iron abundance $<[\mathrm{Fe} / \mathrm{H}]>$ of $\approx 0$ (i.e., the slope is very small, if present). Beyond $R_{\mathrm{G}} \approx 10.0 \mathrm{kpc}$ the mean $<[\mathrm{Fe} / \mathrm{H}]>$ is $\approx-0.3$. This implies a sharp discontinuity at $R_{\mathrm{G}} \approx 10 \mathrm{kpc}$.

Recently, Caputo et al. (2001) reported a similar result. Those authors calibrated $B V I$ data for a large sample of galactic Cepheids (galactocentric distances from 6 to $19 \mathrm{kpc}$ ) as a function of metallicity using non-linear pulsation models. Their results (although not very reliable on a per star basis) suggest that the derived metallicity distribution in the galactic disc can be represented either by a single gradient of $-0.05 \mathrm{dex} \mathrm{kpc}^{-1}$, or by a two-zone distribution with a

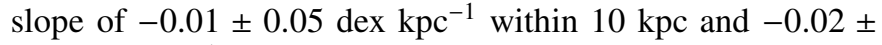
$0.02 \mathrm{dex} \mathrm{kpc}^{-1}$ in the outer region of the galactic disc. In other words, within each region the metallicity gradient is weak to non-existent, while between these regions a significant change of the metallicity/gradient does occur.

These results require an independent check, as well as further specification of the quantitative characteristics of the radial abundance distribution in the outer part of the galactic disc. With this aim we have organized a separate study of distant Cepheids covering the span of distances from 10 to $12 \mathrm{kpc}$. A short description of the observational material is given in the next section.

\section{Observations}

Observations were carried out between 29 November and 2 December of 2001 with the 1.52-m telescope at La Silla (ESO, Chile). The echelle spectrograph FEROS equipped with a $2048 \times 4096$ CCD was used. The resolving power was 48000 . The maximum exposure duration was $60 \mathrm{~min}$. All the spectra have a $S / N$ ratio of about 100 . Only for EE Mon is the $S / N$ lower $(\approx 50$ or less). Table 1 contains some information about our program Cepheids. The columns give the star name, the period $P$ (days), the $V$ magnitude, the exposure times (s), JD, phase, and derived quantities, such as the effective temperature, gravity and microturbulent velocity (see next section).

\section{Atmosphere parameters, abundances and distances}

In Paper I the pertinent details concerning the determination of atmospheric parameters and elemental abundances can be found along with a description of our method of estimating the galactocentric distances. The same methods were used in Paper II and here.

To estimate the heliocentric distances of program Cepheids we used the "absolute magnitude - pulsational period" relation of Gieren et al. (1998). $E(B-V),\langle B-V>$, mean visual magnitudes and pulsational periods are from Fernie et al. (1995), see Table 4. We use for the interstellar absorption $A_{\mathrm{v}}$ an expression from Laney \& Stobie (1993).

For s-Cepheids (DCEPS type) the observed periods are those of the first overtone (see, e.g. Christensen-Dalsgaard \& Petersen 1995). Therefore, following Papers I and II for these stars the corresponding periods of the unexcited fundamental

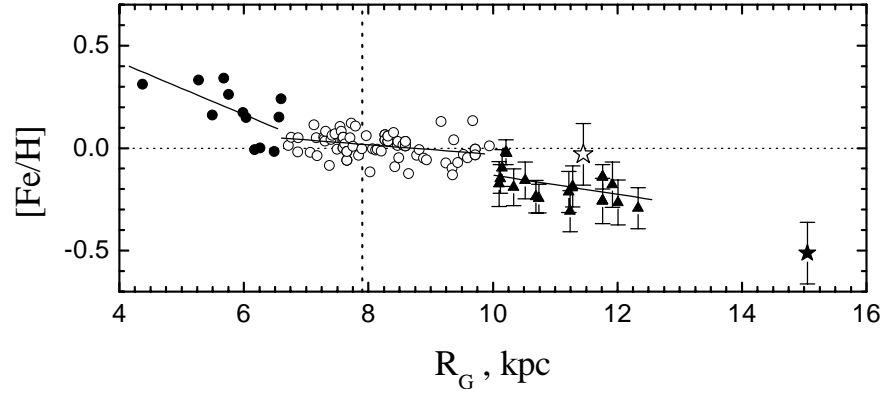

Fig. 1. The radial distribution of the iron abundance. Open circles - the data from Paper I, black circles - the data from Paper II, black triangles - present results. 1- $\sigma$ interval is indicated for the stars from the present study (the error of the mean is of the order of 0.01). The position of EE Mon is indicated by filled asterisk, and TZ Monby open asterisk. The Sun is marked by the intersection of the dashed lines.

mode were found using the ratio $P_{1} / P_{0} \approx 0.72$, and these periods were then used to estimate the absolute magnitudes.

In Tables 1-4 we list the adopted atmospheric parameters of our program stars, derived elemental abundances and distance estimates, together with other (hopefully) useful data. Note that the abundances derived from the lines of different ions are given in the Appendix (Table A1) along with statistical information about the abundances. The abundances of some elements (e.g. N, Sr, Ru, Pr) listed in the Appendix were not used in the present gradient consideration, since the corresponding abundances of these elements were derived only for a minority of the program stars from Papers I and II.

\section{Radial abundance distributions: From inner to outer disc}

Using the results presented in Tables $2-4$ one can construct the radial abundance distribution in the outer disc. To make the picture on galactic abundance gradients as complete as possible, we plotted data from the present paper together with those from Papers I and II (for the sake of clarity the error bars are shown only for the new sample). Figures 1-5 display the derived dependencies between the abundances of 25 chemical elements and galactocentric distances. As the iron abundances are the most reliable we will concentrate our discussion on the iron gradient $\mathrm{d}[\mathrm{Fe} / \mathrm{H}] / \mathrm{d} R_{\mathrm{G}}$.

\section{Discussion}

\subsection{Iron abundance gradient}

The specific aim of this paper is to extend the current work to greater distances towards the galactic anticenter, and to search for possible irregularities in the abundance distributions. Distances can be separated into three zones: the inner part of the galactic disk (gradient $\mathrm{d}[\mathrm{Fe} / \mathrm{H}] / \mathrm{d} R_{\mathrm{G}} \approx-0.13 \pm$

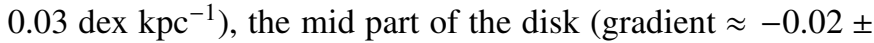
$0.01 \mathrm{dex} \mathrm{kpc}^{-1}$ ), and a piece of outer disc. For the latter we derive a gradient $-0.06 \pm 0.01 \mathrm{dex} \mathrm{kpc}^{-1}$ and a mean 
Table 1. Program stars: details of the observations and atmosphere parameters.

\begin{tabular}{rlrrrcccc}
\hline \hline Star & $P$, day & $V$ & Exp. (s) & JD 2 452 240+ & $\phi$ & $T_{\text {eff }}, \mathrm{K}$ & $\log g$ & $V_{\mathrm{t}}, \mathrm{km} \mathrm{s}^{-1}$ \\
\hline CS Ori & 3.88939 & 11.381 & 3600 & 2.72917 & 0.679 & 6539 & 2.5 & 3.6 \\
AE Tau & 3.89645 & 11.679 & 2400 & 2.65903 & 0.377 & 5981 & 2.3 & 4.0 \\
V504 Mon(s) & 3.907 & 11.814 & 3600 & 3.74583 & 0.714 & 6370 & 2.0 & 3.5 \\
AA Mon & 3.93816 & 12.707 & 2400 & 3.70556 & 0.106 & 6261 & 2.1 & 4.0 \\
EK Mon & 3.95794 & 11.048 & 2400 & 3.63125 & 0.918 & 6001 & 2.0 & 4.5 \\
V495 Mon & 4.096583 & 12.427 & 2400 & 4.83681 & 0.622 & 5591 & 1.6 & 4.0 \\
V508 Mon & 4.133608 & 10.518 & 2400 & 2.76875 & 0.742 & 5566 & 1.6 & 4.0 \\
VW Pup & 4.28537 & 11.365 & 3600 & 4.66250 & 0.617 & 5586 & 1.9 & 4.6 \\
EE Mon & 4.80896 & 12.941 & 3600 & 5.66180 & 0.209 & 6134 & 1.8 & 4.5 \\
XX Mon & 5.456473 & 11.898 & 3600 & 4.79792 & 0.704 & 5533 & 1.7 & 4.2 \\
WW Pup & 5.516724 & 10.554 & 3000 & 4.70833 & 0.722 & 5550 & 1.6 & 4.2 \\
TZ Mon & 7.428014 & 10.761 & 2400 & 3.66944 & 0.817 & 5008 & 2.2 & 1.5 \\
AC Mon & 8.01425 & 10.067 & 2000 & 5.74167 & 0.081 & 6075 & 1.9 & 4.8 \\
TX Mon & 8.70173 & 10.960 & 2000 & 2.80417 & 0.122 & 5871 & 1.8 & 4.9 \\
HW Pup & 13.454 & 12.050 & 3600 & 4.75000 & 0.389 & 5787 & 1.3 & 3.8 \\
AD Pup & 13.5940 & 9.863 & 2400 & 3.78611 & 0.832 & 5124 & 0.9 & 5.1 \\
BN Pup & 13.6731 & 9.882 & 2400 & 3.81944 & 0.396 & 5105 & 1.0 & 3.5 \\
SV Mon & 15.23278 & 8.219 & 1800 & 2.69236 & 0.559 & 4924 & 1.1 & 4.5 \\
VZ Pup & 23.1710 & 9.621 & 1800 & 2.81944 & 0.980 & 6070 & 2.2 & 4.7 \\
\hline
\end{tabular}

For V504 Mon (classified as s-Cepheid) the fundamentalized period is given $\left(P_{0}=P_{1} / 0.71\right), P($ observ $)=P_{1}=2.77405$.

Table 2. Averaged relative-to-solar elemental abundance for program Cepheids: C-Mn.

\begin{tabular}{lrrrrrrrrrrrrrr}
\hline \hline Star & $\mathrm{C}$ & $\mathrm{N}$ & $\mathrm{O}$ & $\mathrm{Na}$ & $\mathrm{Mg}$ & $\mathrm{Al}$ & $\mathrm{Si}$ & $\mathrm{S}$ & $\mathrm{Ca}$ & $\mathrm{Sc}$ & $\mathrm{Ti}$ & $\mathrm{V}$ & $\mathrm{Cr}$ & $\mathrm{Mn}$ \\
\hline CS Ori & -0.58 & -0.12 & -0.61 & 0.05 & -0.40 & -0.31 & -0.16 & -0.32 & -0.20 & -0.11 & -0.23 & - & -0.24 & -0.43 \\
AE Tau & -0.48 & 0.07 & -0.17 & -0.10 & -0.36 & -0.30 & -0.18 & -0.33 & -0.25 & -0.08 & -0.06 & -0.21 & -0.12 & -0.41 \\
V504 Mon & -0.53 & 0.14 & -0.35 & -0.08 & -0.45 & -0.13 & -0.17 & -0.23 & -0.21 & -0.33 & -0.28 & -0.11 & -0.30 & -0.38 \\
AA Mon & -0.49 & 0.35 & -0.19 & 0.04 & -0.22 & -0.14 & -0.12 & -0.12 & -0.26 & -0.21 & -0.23 & 0.02 & -0.26 & -0.29 \\
EK Mon & -0.42 & 0.26 & -0.28 & 0.04 & -0.18 & -0.04 & -0.05 & -0.15 & -0.10 & -0.05 & -0.18 & -0.10 & -0.15 & -0.19 \\
V495 Mon & -0.77 & -0.12 & -0.09 & 0.00 & -0.28 & -0.25 & -0.18 & -0.07 & -0.29 & -0.34 & -0.29 & -0.28 & -0.32 & -0.39 \\
V508 Mon & -0.77 & 0.07 & -0.54 & -0.01 & -0.28 & -0.15 & -0.18 & -0.19 & -0.22 & -0.21 & -0.13 & -0.22 & -0.22 & -0.41 \\
VW Pup & -0.48 & -0.12 & 0.19 & 0.02 & -0.31 & -0.21 & -0.16 & -0.29 & -0.23 & -0.19 & -0.28 & -0.31 & -0.07 & -0.37 \\
EE Mon & -0.48 & 0.14 & - & -0.20 & -0.60 & - & -0.35 & -0.56 & -0.50 & -0.42 & -0.26 & -0.41 & -0.35 & -0.66 \\
XX Mon & -0.52 & 0.15 & 0.06 & 0.02 & - & 0.13 & -0.18 & -0.21 & -0.18 & -0.10 & -0.23 & -0.06 & -0.13 & -0.29 \\
WW Pup & -0.64 & -0.16 & - & 0.01 & -0.33 & -0.12 & -0.14 & -0.20 & -0.22 & -0.31 & -0.25 & -0.19 & -0.20 & -0.31 \\
TZ Mon & -0.53 & 0.26 & 0.28 & 0.15 & -0.05 & 0.05 & 0.00 & 0.20 & 0.06 & -0.27 & -0.08 & -0.08 & -0.05 & 0.41 \\
AC Mon & -0.52 & 0.23 & -0.25 & 0.05 & - & -0.36 & -0.08 & -0.21 & -0.13 & -0.21 & -0.19 & -0.23 & -0.24 & -0.25 \\
TX Mon & -0.41 & 0.29 & -0.16 & -0.08 & -0.26 & -0.07 & -0.09 & -0.17 & -0.24 & -0.16 & -0.17 & -0.28 & -0.16 & -0.25 \\
HW Pup & -0.56 & 0.04 & -0.29 & -0.20 & -0.52 & -0.16 & -0.19 & -0.29 & -0.27 & -0.30 & -0.25 & -0.33 & -0.29 & -0.28 \\
AD Pup & -0.77 & 0.49 & - & 0.06 & - & 0.00 & -0.19 & -0.13 & -0.17 & -0.04 & -0.22 & -0.32 & -0.38 & -0.33 \\
BN Pup & -0.45 & 0.55 & -0.06 & 0.09 & 0.21 & 0.04 & -0.01 & 0.19 & 0.04 & -0.02 & -0.05 & -0.15 & 0.07 & -0.02 \\
SV Mon & -0.81 & 0.22 & 0.21 & 0.10 & -0.48 & -0.04 & -0.02 & 0.24 & -0.13 & -0.07 & -0.10 & -0.19 & -0.05 & 0.03 \\
VZ Pup & -0.83 & 0.02 & -0.10 & 0.00 & -0.15 & -0.15 & -0.09 & -0.22 & -0.12 & -0.16 & -0.26 & -0.01 & -0.18 & -0.28 \\
\hline
\end{tabular}

$[\mathrm{Fe} / \mathrm{H}] \approx-0.19 \pm 0.08$ dex. The gradient for each zone was derived from a least-squares fit using the weighted data (for the weights assigned to the stars of the inner and mid parts of the disc see Papers I and II). Each star of the present sample was assigned unit weight ( $W=1$ ). Two stars (TV Cam and YZ Aur) from the outer zone were excluded from the present determination. For those Cepheids we have analyzed only photographic spectra (see Paper I), therefore we consider the abundance results (only iron) for TV Cam and YZ Aur as being much less reliable than for other stars where we have the CCD spectra. We also excluded from the statistics the very distant Cepheid EE Mon, and the anomalous Cepheid TZ Mon (about the latter see below). The former will be included in the statistics consideration after we sample the region of galactocentric distances from $12 \mathrm{kpc}$ to $15 \mathrm{kpc}$ (next papers of this series).

The transition zone at $10 \mathrm{kpc}$ can be easily identified in Fig. 1. After this point the metallicity drops by approximately 0.2 dex. All the stars in the bin beyond $10 \mathrm{kpc}$ are irondeficient. The same result is seen in Figs. 3a, b of Twarog et al. (1997) which shows their open cluster metallicity values as a function of galactocentric radius. It should be noted that the sample of open clusters used by Twarog et al. consists of the clusters with ages spanning from 1 to 5 Gyr. Thus, the youngest clusters used for the gradient study are approximately 10 times 
Table 3. Same as Table 2 but for Fe-Gd.

\begin{tabular}{lrrrrrrrrrrrr}
\hline \hline Star & $\mathrm{Fe}$ & $\mathrm{Co}$ & $\mathrm{Ni}$ & $\mathrm{Cu}$ & $\mathrm{Zn}$ & $\mathrm{Y}$ & $\mathrm{Zr}$ & $\mathrm{La}$ & $\mathrm{Ce}$ & $\mathrm{Nd}$ & $\mathrm{Eu}$ & $\mathrm{Gd}$ \\
\hline CS Ori & -0.26 & - & -0.25 & 0.19 & - & -0.09 & - & 0.21 & 0.07 & -0.17 & -0.03 & - \\
AE Tau & -0.19 & -0.45 & -0.22 & -0.14 & - & 0.08 & -0.18 & 0.32 & -0.27 & -0.08 & 0.11 & - \\
V504 Mon & -0.31 & - & -0.36 & 0.20 & - & -0.17 & -0.24 & - & -0.26 & -0.30 & -0.15 & - \\
AA Mon & -0.21 & - & -0.30 & 0.29 & - & 0.07 & - & - & 0.03 & -0.11 & -0.32 & - \\
EK Mon & -0.10 & -0.06 & -0.20 & -0.96 & - & -0.07 & 0.05 & - & -0.22 & 0.06 & 0.31 & - \\
V495 Mon & -0.26 & -0.25 & -0.36 & -0.31 & - & -0.10 & -0.44 & 0.05 & 0.04 & -0.14 & -0.17 & - \\
V508 Mon & -0.25 & -0.35 & -0.35 & -0.30 & 0.11 & -0.17 & -0.16 & 0.06 & -0.17 & -0.24 & -0.27 & -0.14 \\
VW Pup & -0.19 & -0.26 & -0.17 & -0.64 & - & -0.09 & - & 0.19 & 0.08 & -0.06 & -0.08 & - \\
EE Mon & -0.51 & - & -0.58 & - & - & -0.40 & - & - & -0.27 & -0.23 & - & - \\
XX Mon & -0.18 & -0.27 & -0.27 & -0.22 & - & 0.00 & -0.25 & 0.06 & -0.05 & 0.01 & 0.04 & - \\
WW Pup & -0.18 & - & -0.21 & -0.34 & -0.01 & 0.04 & - & 0.15 & 0.00 & -0.04 & -0.09 & - \\
TZ Mon & -0.03 & -0.16 & -0.09 & 0.17 & 0.26 & -0.21 & -0.22 & 0.04 & -0.26 & -0.25 & -0.01 & - \\
AC Mon & -0.22 & - & -0.35 & -0.80 & - & -0.12 & - & 0.13 & -0.20 & -0.12 & 0.18 & - \\
TX Mon & -0.14 & -0.24 & -0.20 & -0.04 & 0.15 & 0.06 & -0.27 & 0.23 & 0.10 & -0.10 & 0.07 & -0.23 \\
HW Pup & -0.29 & -0.18 & -0.35 & -0.32 & - & -0.13 & - & - & -0.13 & -0.15 & -0.11 & - \\
AD Pup & -0.24 & -0.48 & -0.37 & -0.47 & - & -0.11 & -0.20 & 0.27 & -0.14 & -0.12 & -0.06 & -0.03 \\
BN Pup & 0.01 & -0.23 & -0.03 & -0.24 & - & 0.23 & -0.17 & 0.30 & 0.00 & 0.03 & 0.07 & - \\
SV Mon & 0.00 & -0.24 & -0.08 & -0.42 & - & 0.17 & 0.00 & 0.30 & 0.08 & 0.16 & 0.08 & 0.12 \\
VZ Pup & -0.16 & - & -0.28 & 0.10 & - & -0.06 & - & - & -0.11 & 0.02 & 0.02 & 0.32 \\
\hline
\end{tabular}

Table 4. Physical and positional characteristics for program Cepheids.

\begin{tabular}{rrrrrrrrrr}
\hline \hline Star & $P, \mathrm{~d}$ & $\langle(B-V)>$ & $E(B-V)$ & \multicolumn{1}{c}{$M_{\mathrm{v}}$} & $d, \mathrm{pc}$ & $l$ & $b$ & $R_{\mathrm{G}}, \mathrm{kpc}$ & {$[\mathrm{Fe} / \mathrm{H}]$} \\
\hline CS Ori & 3.88939 & 0.924 & 0.402 & -2.93 & 3997.6 & 197.97 & -4.51 & 11.76 & -0.26 \\
AE Tau & 3.89645 & 1.129 & 0.604 & -2.93 & 3389.8 & 181.03 & -3.52 & 11.28 & -0.19 \\
V504 Mon & 3.907 & 1.036 & 0.565 & -2.93 & 3845.9 & 215.69 & -3.29 & 11.24 & -0.31 \\
AA Mon & 3.93816 & 1.409 & 0.832 & -2.94 & 3860.2 & 217.03 & -0.43 & 11.22 & -0.21 \\
EK Mon & 3.95794 & 1.195 & 0.584 & -2.95 & 2618.1 & 215.27 & -0.82 & 10.15 & -0.10 \\
V495 Mon & 4.096583 & 1.241 & 0.640 & -2.99 & 4630.0 & 213.81 & -4.49 & 12.01 & -0.26 \\
V508 Mon & 4.133608 & 0.898 & 0.323 & -3.00 & 3120.1 & 208.91 & 0.86 & 10.74 & -0.25 \\
VW Pup & 4.28537 & 1.065 & 0.514 & -3.04 & 3533.7 & 235.37 & -0.62 & 10.33 & -0.19 \\
EE Mon & 4.80896 & 0.966 & 0.488 & -3.18 & 8130.6 & 219.97 & -3.77 & 15.05 & -0.51 \\
XX Mon & 5.456473 & 1.139 & 0.596 & -3.33 & 4566.6 & 215.52 & -1.11 & 11.92 & -0.18 \\
WW Pup & 5.516724 & 0.874 & 0.398 & -3.35 & 3342.7 & 237.38 & 0.97 & 10.10 & -0.18 \\
TZ Mon & 7.428014 & 1.116 & 0.441 & -3.71 & 4020.6 & 214.01 & 1.29 & 11.45 & -0.03 \\
AC Mon & 8.01425 & 1.165 & 0.508 & -3.80 & 2754.8 & 221.77 & -1.85 & 10.12 & -0.22 \\
TX Mon & 8.70173 & 1.096 & 0.511 & -3.90 & 4350.8 & 214.14 & -0.78 & 11.76 & -0.14 \\
HW Pup & 13.454 & 1.237 & 0.723 & -4.42 & 6684.1 & 244.77 & 0.78 & 12.33 & -0.29 \\
AD Pup & 13.5940 & 1.049 & 0.330 & -4.43 & 4388.0 & 241.93 & -0.03 & 10.69 & -0.24 \\
BN Pup & 13.6731 & 1.186 & 0.438 & -4.44 & 3762.1 & 247.90 & 1.07 & 9.95 & 0.01 \\
SV Mon & 15.23278 & 1.048 & 0.249 & -4.57 & 2472.3 & 203.74 & -3.67 & 10.21 & 0.00 \\
VZ Pup & 23.1710 & 1.162 & 0.471 & -5.07 & 4263.0 & 243.42 & -3.31 & 10.52 & -0.16 \\
\hline
\end{tabular}

older than Cepheids. By comparing the iron abundance gradient from Cepheids with that from open clusters one would, in principle, estimate how the abundance gradient evolved with time. Nevertheless, in practice it is difficult to realize, because any conclusion will suffer from the rather high uncertainty of the open cluster data. The only we can state confidently is that the discontinuity of the metallicity distribution has really survived over several Gyrs, until now.

Using a linear fit to the $[\mathrm{Fe} / \mathrm{H}]$ data over the entire baseline $(4-12 \mathrm{kpc})$ produces a gradient of $-0.06 \mathrm{dex} \mathrm{kpc}^{-1}$ which is surprisingly close to the canonical literature value of $-0.06 /-0.07 \mathrm{dex} \mathrm{kpc}^{-1}$ (see Papers I and II and references therein).

\subsection{Radial distribution of other species}

In this paper we will not discuss in detail the radial distributions of the elements other than iron. This will be done in a future paper using the whole observed baseline $4-15 \mathrm{kpc}$ after the region of galactocentric distances between 12 and $15 \mathrm{kpc}$ is well sampled.

As is well known, the carbon abundance is altered during the Cepheid evolution, specifically as a result of the first dredge-up. Therefore we do not attach a great significance to the radial distribution obtained for this element. Nevertheless, interestingly enough, the $[\mathrm{C} / \mathrm{H}]-R_{\mathrm{G}}$ dependence is rather clear. Two remarkable features inherent to carbon abundance 

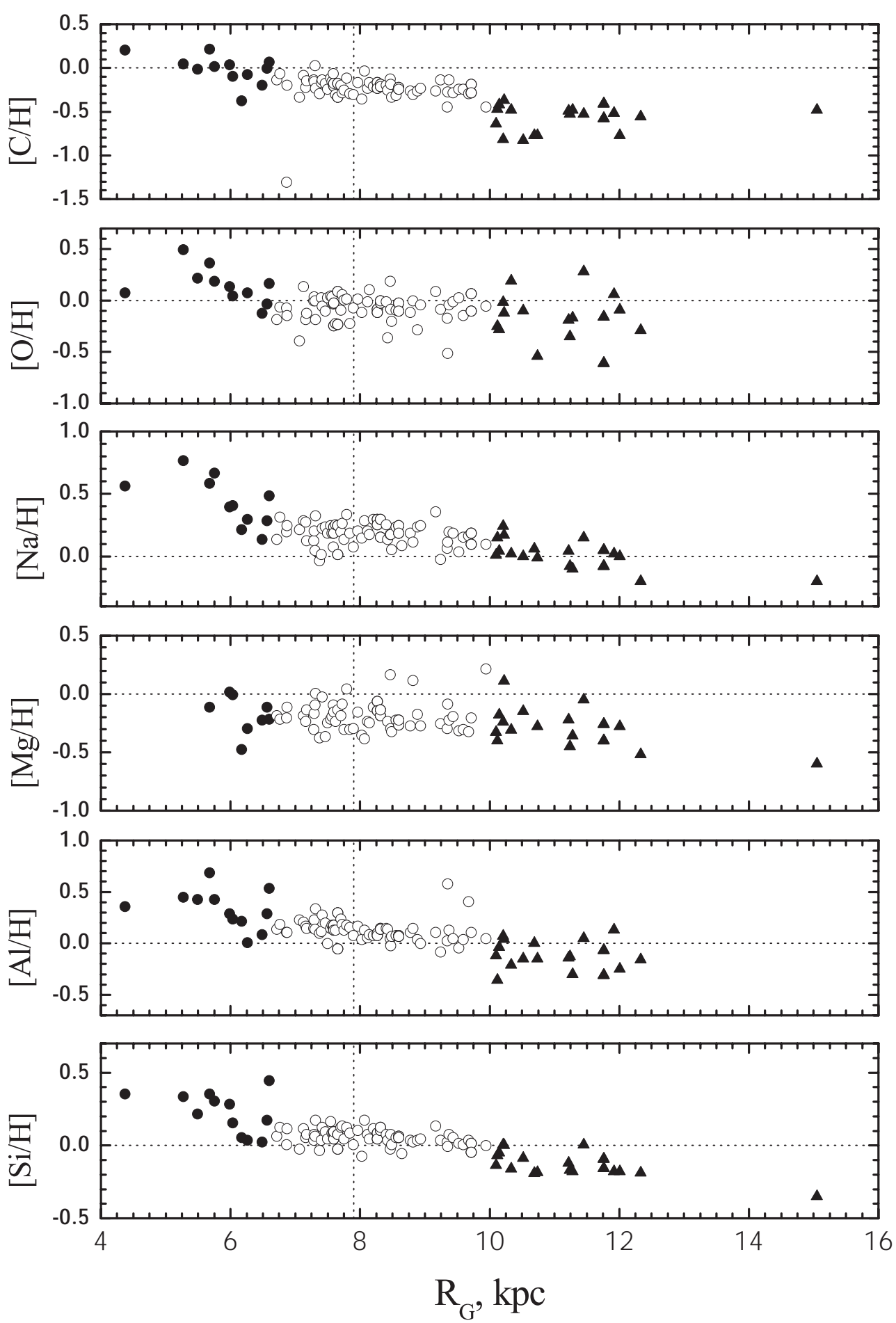

Fig. 2. Same as Fig. 1, but for elements C-Si.

distribution should be stressed. First, the extremely low carbon content in FN Aql (see Paper I and Fig. $2, R_{\mathrm{G}} \approx 6.9 \mathrm{kpc}$ ), and second, the well expressed "dip" in carbon abundance at galactocentric distances near $10 \mathrm{kpc}$. The presence of a coherent behavior in carbon can be interpreted as the original carbon abundances show a similar gradient, and as the mixing event at the first dredge-up modifies all of the abundances by similar amounts; i.e., that the mixing process has similar efficiency (depth) for all stars in this mass range.
We believe that rather large scatter in the radial distribution of oxygen abundance at distances $10-12 \mathrm{kpc}$ is probably caused by some unreliability in oxygen abundance derived for distant Cepheids. This scatter probably does not reflect the real inhomogeneity of oxygen abundance in the interstellar media of the outer disc. The same can be said about elements such as Sc, $\mathrm{Cu}, \mathrm{Zn}$ (only a few spectral lines for each of these elements including $\mathrm{O}$ were analyzed). Those elements whose abundances 

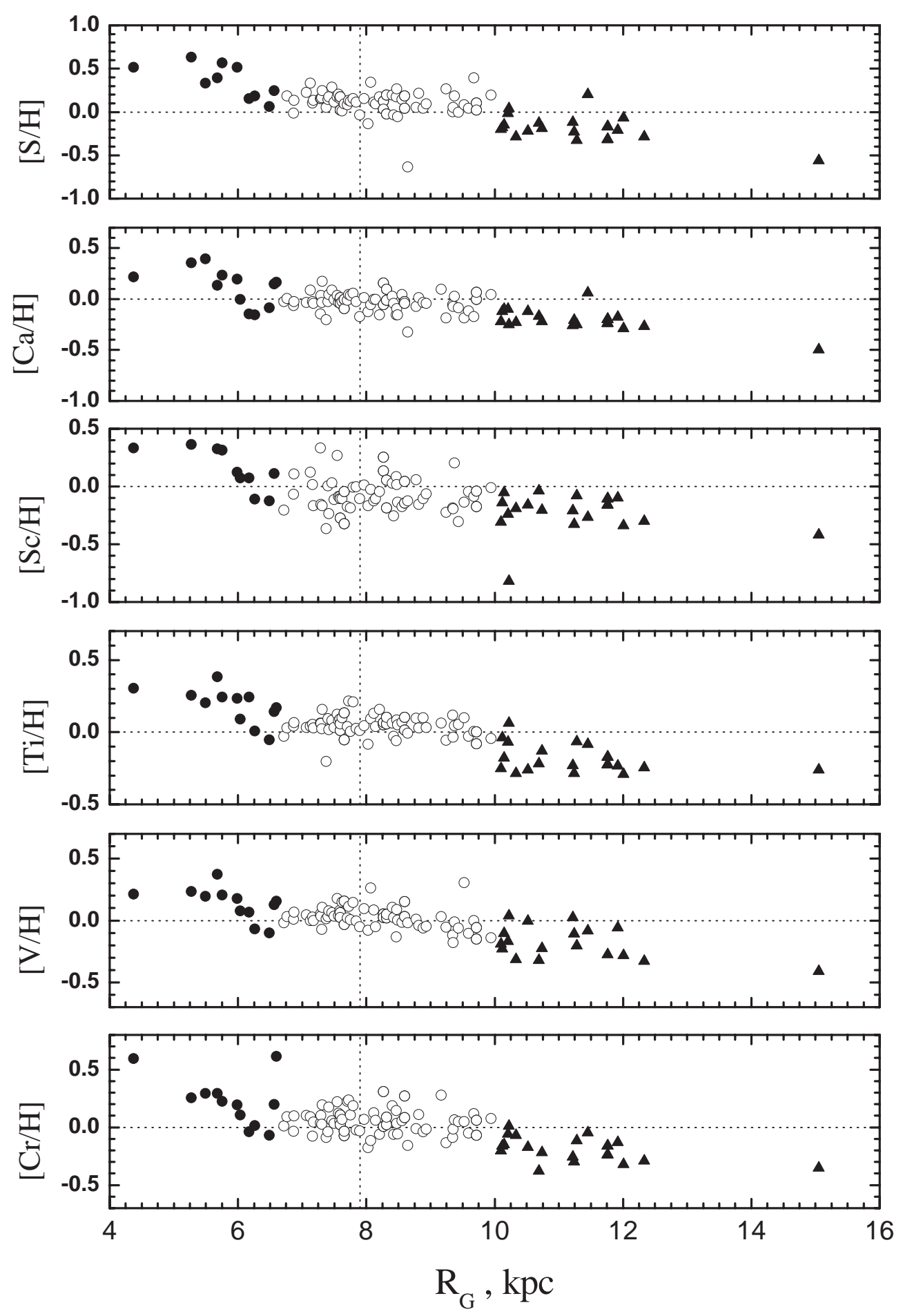

Fig. 3. Same as Fig. 1, but for elements $\mathrm{S}-\mathrm{Cr}$.

were based on the analysis of a significant number of lines (e.g. $\mathrm{Si}, \mathrm{Ti}, \mathrm{V}, \mathrm{Ni}$ ), show rather tight distributions.

The step distribution and apparent discontinuity (similar to iron) is also seen for $\mathrm{Si}, \mathrm{Ti}, \mathrm{Mn}, \mathrm{Co}, \mathrm{Ni}, \mathrm{Zr}$ and $\mathrm{Nd}$.

\subsection{A possible explanation for the observed features}

If we consider the ensemble of our results, paying special attention to the elements for which the data present smaller scatter because they present a large number of lines in the spectra, like $\mathrm{Si}, \mathrm{Ti}, \mathrm{V}, \mathrm{Ni}$, in addition to $\mathrm{Fe}$ and $\mathrm{C}$, it is very clear that the metallicity distribution is flat between about 6 and $10 \mathrm{kpc}$, and that there is a discontinuity of about -0.2 dex for $R_{\mathrm{G}}>10 \mathrm{kpc}$, and perhaps a minimum at about $10.5-11.0 \mathrm{kpc}$ which can be traced from some distributions (let us recall that we adopt $R_{\odot}=7.9 \mathrm{kpc}$ ). This is a new result, although the discontinuity at about $10 \mathrm{kpc}$ was already observed by Twarog et al. (1997), in a sample of open clusters. An attractive explanation for the 

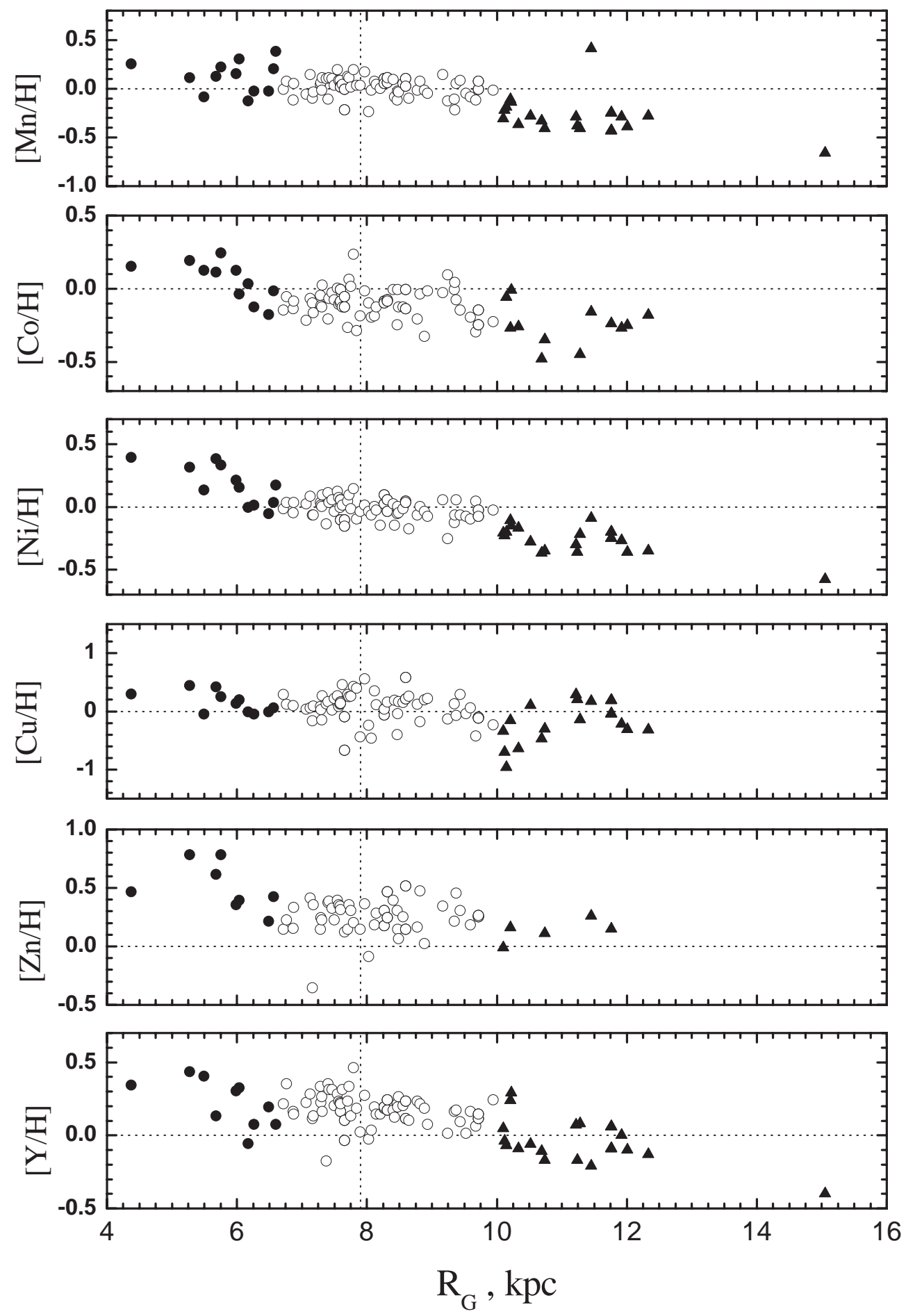

Fig. 4. Same as Fig. 1, but for elements Mn-Y.

minimum at $10.5 \mathrm{kpc}$ (if it truly exists) is the minimum of star formation rate that is expected at co-rotation, as mentioned in the Introduction.

Recently Mishurov et al. (2002) produced a detailed model of chemical evolution of the galactic disc, taking into account the effect of co-rotation, to explain our data presented in Papers I and II. The new data presented in this paper, suggest that the model of Mishurov et al. (2002) is basically correct, but that the co-rotation radius should be slightly shifted to about $10.5 \mathrm{kpc}$. The discontinuity in the metallicity distribution at $10 \mathrm{kpc}$ is possibly explained by the gap in the gas density distribution that is associated with co-rotation (see Lépine et al. 2001). If we divide the Galactic disc in a large number of concentric rings, the gas from neighbouring rings tends to mix due to supernova explosions, stellar winds, cloud collisions, etc., that do not respect the frontiers between concentric rings. This mixing is equivalent to a diffusion term, and tends to smooth out metallicity gradients in the gas (and therefore, in recently 

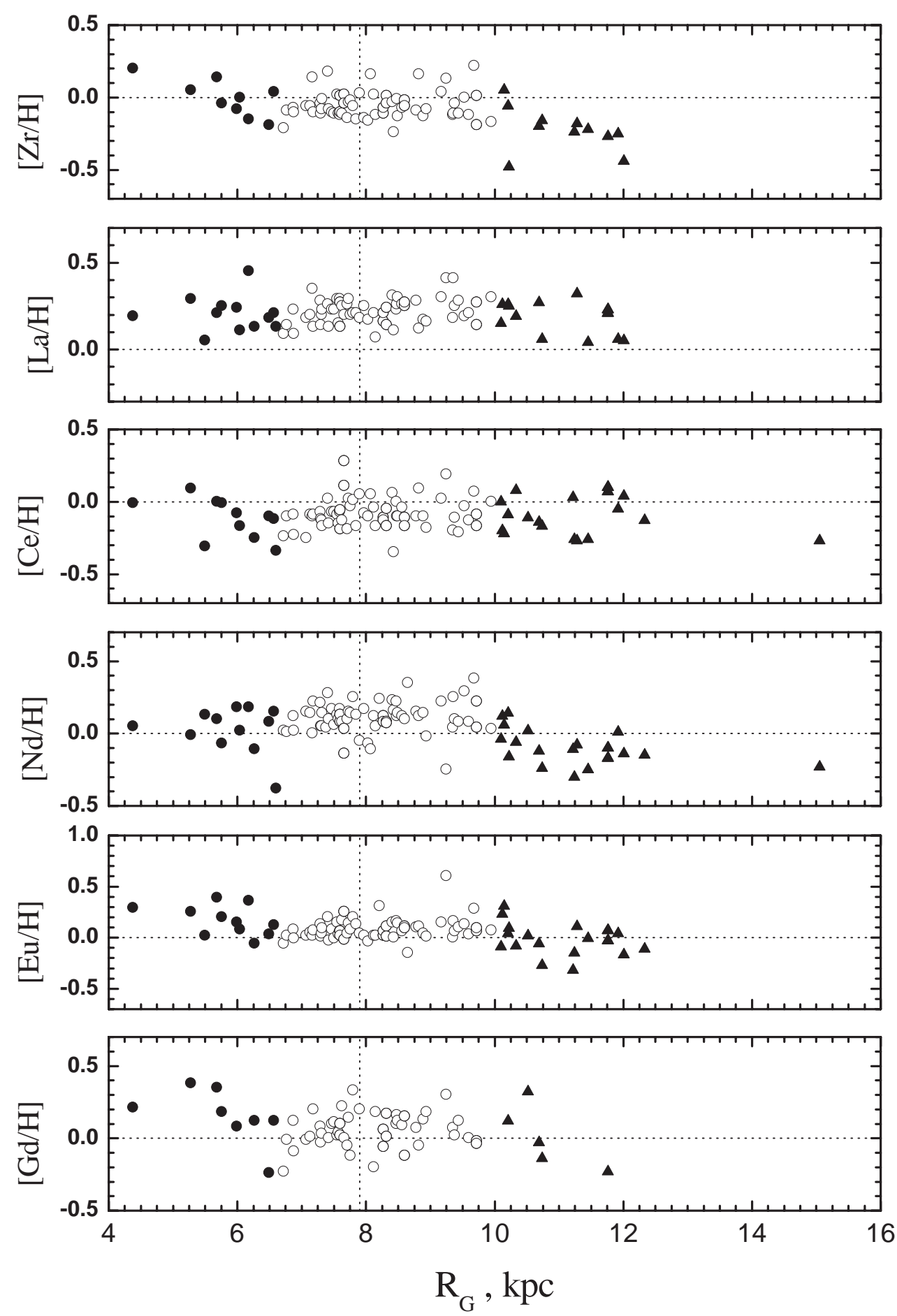

Fig. 5. Same as Fig. 1, but for elements $\mathrm{Zr}-\mathrm{Gd}$.

formed stars). However, the gas density gap associated with corotation, which is observed in the $21 \mathrm{~cm}$ hydrogen line as discussed by Lépine et al. (2001) is possibly a barrier that avoids contact between the gas at $R_{\mathrm{G}}>R_{\mathrm{c}}$ and $R_{\mathrm{G}}<R_{\mathrm{c}}$ and allows the existence of two distinct zones.

\subsection{Some notes about TZ Mon}

This star differs from other Cepheids by its very low $V_{\mathrm{t}}$ value (1.5 $\mathrm{km} \mathrm{s}^{-1}$, see Table 1) which is not appropriate for supergiants. Nevertheless, only by using this small value can one avoid any dependence between iron abundance from individual iron lines and their equivalent widths. With the adopted microturbulent velocity this distant Cepheid shows solar-like abundances for the great majority of elements. For Mn we detected a strong overabundance. Another interesting feature, which is seen in the TZ Mon spectrum, is the $6707 \AA \mathrm{Li} \mathrm{I}$ line. Normally this line is not present in supergiant spectra. The equivalent width of $W=10 \mathrm{~m} \AA$ results in an absolute lithium abundance for TZ Mon of about 0.84 . This is consistent with $\mathrm{Li}$ 
abundances previously determined for some non-variable $\mathrm{G} / \mathrm{K}$ supergiants by Luck (1977).

Acknowledgements. This work was supported in part by a grant to SMA from FAPESP São Paulo (visiting professor fellowship No. 2000/06587-3), and ESO Garching (visitor programme). SMA is very grateful to Drs. B Barbuy, M. Spite, F. Spite and S. D'Odorico for their personal assistance. He would like also to thank the staff of ESO La Silla for the provision of excellent conditions during his observations.

The authors are indebted to the referee, Dr. B.W. Carney, for important comments which allowed us to improve the fist version of this paper.

\section{References}

Amaral, L. H., \& Lépine, J. R. D. 1997, MNRAS, 286, 885

Andrievsky, S. M., Kovtyukh, V. V., Luck, R. E., et al. 2002a, A\&A, 381,32
Andrievsky, S. M., Bersier, D., Kovtyukh, V. V., et al. 2002b, A\&A, 384,140

Caputo, F., Marconi, M., Musella, I., \& Pont, F. 2001, A\&A, 372, 544

Christensen-Dalsgaard, Petersen, J. O. 1995, A\&A, 299, L17

Fernie, J. D., Evans, N. R., Beattie, B., \& Seager, S. 1995, IBVS, 4148,1

Friel, E. D. 1995, ARA\&A, 33, 381

Gieren, W. P., Fouqué, P., \& Gómez, M. 1998, ApJ, 496, 17

Janes, K. A. 1979, ApJS, 39, 135

Laney, C. D., \& Stobie, R. S. 1993, MNRAS, 263, 291

Lépine, J. R. D., Mishurov, Yu. N., \& Dedikov, S. Yu. 2001, ApJ, 546, 234

Luck, R. E. 1977, ApJ, 218, 752

Mishurov, Yu. N., Lépine, J. R. D., \& Acharova, I. A. 2002 [astro-ph/0203458]

Panagia, N., \& Tosi, M. 1981, A\&A, 96, 306

Sevenster, M. N. 1999a, MNRAS, 310, 629

Sevenster, M. N. 1999b, ApSS, 265, 377

Twarog, B. A., Ashman, K. M., \& Antony-Twarog, B. J. 1997, AJ, 114,2556 